Revista de Comunicación y Salud, 2020, Vol. 10, no 2, pp. 569-591

Editado por Cátedra de Comunicación y Salud

ISSN: 2173-1675

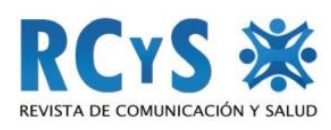

Enviado $13 / 08 / 2020$

Aprobado 17/09/2020

\title{
PERIODISMO, COMUNICACIÓN INSTITUCIONAL Y TRANSPARENCIA: APRENDIZAJES DE LA CRISIS SANITARIA DEL COVID-19
}

\author{
Journalism, institutional communication and transparency: lessons from the \\ covid-19 health crisis
}

\author{
Javier Sierra Rodríguez ${ }^{1}$ \\ Universidad de Murcia. España \\ javier.sierra@um.es
}

\section{Resumen}

Introducción: El objetivo del artículo es extraer aprendizajes para la modificación y mejora de las normas reguladoras de la transparencia en España ante situaciones excepcionales como el estado de alarma, de modo que sirva a finalidades como facilitar la rendición de cuentas y a la labor de los profesionales del periodismo. Metodología: para ello, se realiza un análisis de la controversia sobre la falta de transparencia gubernamental en el contexto del estado de alarma, realizando una reconstrucción de los hitos y circunstancias acontecidas entre los meses de marzo y mayo de 2020 con relación a la transparencia gubernamental del Estado, combinando para ello diversas fuentes de información como estadísticas, datos de encuestas del CIS, noticias de prensa, informes jurídicos y bibliografía. Resultados: el análisis confirma las insuficiencias de la normativa de transparencia detectadas en la literatura por expertos y periodistas. Entre ellas, la falta de un reconocimiento del acceso a la información pública como derecho fundamental, el exceso de los plazos para obtener respuesta a solicitudes de acceso, la escasa utilidad para desarrollar el periodismo de datos o la debilidad del Consejo de la Transparencia y Buen Gobierno, aparte de una falta general de previsiones para hacer eficaz la rendición de cuentas en materia sanitaria y ante la utilización de los contratos de emergencia.

Palabras clave: Covid-19; transparencia; libertad de información; periodismo; derecho de acceso; datos sanitarios; contratos de emergencia.

\footnotetext{
1 Autor para correspondencia: Javier Sierra Rodríguez javier.sierra@um.es : Politólogo y Doctor en Derecho Público. Trabaja profesionalmente en investigación social y es profesor de varias universidades españolas con una amplia producción investigadora en materia de transparencia y participación.
} 
Periodismo, comunicación institucional y transparencia: aprendizajes de la crisis sanitaria del COVID-19

\begin{abstract}
Introduction: The objective of the article is to extract learnings for the modification and improvement of the regulatory norms of transparency in Spain, in exceptional situations such as the state of alarm, so that it serves purposes such as to facilitate the accountability and work of journalism professionals. Methodology: For this, an analysis of the controversy on the lack of government transparency in the context of the state of alarm is carried out,, performing a reconstruction of the milestones and circumstances that occurred between the months of March and May 2020 in relation to the State's government transparency, combining various sources of information such as statistics, CIS survey data, press news, legal reports and bibliography. Results: the analysis confirms the insufficiencies of the transparency regulations detected in the literature by experts and journalists. Among them, the lack of recognition of access to public information as a fundamental right, the excess of deadlines to respond to requests for access, the limited use to develop data journalism or the weakness of the Council of Transparency and Good Governance, apart from a general lack of provisions to make accountability effective in health and the use of emergency contracts.
\end{abstract}

Keywords: Covid-19; Transparency; Freedom of information; Journalism; Right of access; Health data; Emergency contracts.

\title{
Cómo citar el artículo
}

Sierra Rodríguez, J. (2020). Periodismo, comunicación institucional y transparencia: aprendizajes de la crisis sanitaria del COVID-19. Revista de Comunicación y Salud, 10 (2), 569-591. doi: https://doi.org/10.35669/rcys.2020.10(2).569-591

\section{CRISIS DEL COVID Y TRANSPARENCIA}

Durante los meses de abril y mayo de 2020 y en plena crisis por el COVID-19 afloró una controversia sobre la falta de transparencia de la actividad gubernamental, debido a la confluencia de factores como la filtración de preguntas en las ruedas de prensa del Gobierno y la paralización o demora de los mecanismos que permiten acceder a información pública.

En un contexto como el de una crisis sanitaria en el marco del estado de alarma, la información gubernamental y oficial sin restricciones adquiere una mayor importancia por el aumento de la demanda ciudadana, y como fórmula para ejercer un nivel mínimo de rendición de cuentas sobre la actividad gubernamental.

La labor de los medios de comunicación contribuye al ejercicio de ese de control, que se complementa con los mecanismos de transparencia que existen a partir de la Ley de Transparencia, Acceso a la Información Pública y Buen Gobierno (Ley 19/2013, de 9 de diciembre), y de las obligaciones otras leyes sectoriales, de los cuáles se benefician los 
Periodismo, comunicación institucional y transparencia: aprendizajes de la crisis sanitaria del COVID-19

ciudadanos en general, y dentro de ellos, los periodistas porque pueden acudir a las herramientas que habilitan para el desarrollo de su labor.

Desde la publicación de la Ley de Transparencia en 2013 se ha transitado desde unas altas expectativas respecto a las posibilidades de la transparencia, a apreciar de manera general que existen muchos retos pendientes (Sánchez de Diego y Sierra Rodríguez, 2020). En el ámbito del periodismo ha sucedido lo mismo, apuntalando hoy en día una percepción generalizada de insuficiencia del marco normativo (Díez Garrido y Campos Domínguez, 2018; Rubio Jiménez, 2017; La Rosa Barrolleta y Sandoval Martín, 2016).

Este artículo tiene por objeto profundizar en el análisis de esas insuficiencias de la regulación de la transparencia con relación al periodismo en el contexto de la crisis sanitaria originada por el COVID-19, a la luz de los acontecimientos que se produjeron desde que se declarase el estado de alarma - a mediados del mes de marzo de 2020 - hasta el inicio del mes de junio de 2020, fecha a partir de la cual se restablecieron algunos procedimientos para permitir el acceso a la información pública.

Para ello, se recurre al tratamiento de este periodo como un estudio de caso y se profundiza en su reconstrucción a través de la utilización de fuentes de diverso tipo como datos del CIS, estadísticas de solicitudes de acceso a la información pública e informes de la administración pública, aparte del análisis bibliográfico, normativo y jurisprudencial.

\section{TRANSPARENCIA, PERIODISMO E INFORMACIÓN EN ÉPOCA DE CRISIS}

El acercamiento a la literatura de referencia para este artículo se desarrolla en torno a tres temáticas interrelacionadas: la noción y funciones de la transparencia, su proyección con relación al periodismo, y la singularidad de la información pública ante la crisis sanitaria.

\subsection{La noción de transparencia y sus funciones}

La transparencia puede ser entendida como un ideal, un principio o bien describirla en el marco de nuestra regulación actual. Para Sánchez de Diego (2016: 307) viene a ser "una cualidad del sistema informativo de las entidades públicas". Por otra parte, Ramírez Alujas (2010: 24 y 25) nos acerca a la descripción de un gobierno transparente como aquel que "proporciona información sobre lo que está haciendo, pone a disposición sus fuentes y bases de datos, y publica los planes de actuación por los que puede ser considerado responsable frente a la sociedad. Ello fomenta y promueve la rendición de cuentas ante la ciudadanía y un permanente control social, al mismo tiempo que refuerza la confianza, la cohesión social y el fortalecimiento institucional".

A través de la regulación de la Ley de Transparencia de 2013 (LTBG) también se puede entender como un principio de actuación de las administraciones públicas, cuyas

Revista de Comunicación y Salud, 2020, Vol. 10, no 2, 569-591. 
Periodismo, comunicación institucional y transparencia: aprendizajes de la crisis sanitaria del COVID-19

manifestaciones principales son el derecho de acceso y la publicidad activa (Piñar Mañas, 2014: 5). El derecho de acceso permite a los ciudadanos solicitar información en poder de las administraciones públicas, aunque está sujeta a límites derivados de materias concretas: seguridad nacional, defensa, relaciones exteriores, seguridad pública, etc. También existe un límite derivado de la afectación a derechos de terceros y en concreto a la protección de datos de carácter personal. Aparte, hay una serie de causas de inadmisión de las solicitudes, ante información en curso de elaboración o publicación, información auxiliar o de apoyo, que precise reelaboración, entre otras. Las causas de denegación y de inadmisión han sido criticadas de manera recurrente porque permiten interpretaciones abusivas que terminen por mermar el derecho de acceso (Moretón Toquero, 2014). Es cierto que se pueden recurrir las denegaciones de acceso por estas causas ante los Consejos de Transparencia, y que éstos adoptan una postura favorable a la transparencia (Barredo Rodríguez, 2017; Mir Puigpelat, 2017), pero de todos modos, esto tiene como consecuencia que se dilaten los plazos para obtener cualquier resolución favorable.

Por otra parte, la publicidad activa se corresponde con la publicación de información a través de las páginas y portales gubernamentales en cumplimiento de las obligaciones que establece la ley. En concreto, se trata de un catálogo de información que debe ser publicada y que se categoriza como información institucional, organizativa y de planificación; de relevancia jurídica (normas, proyectos de normas y memorias e informes); e información económica, presupuestaria y estadística.

De este modo, la regulación de la transparencia -aunque contenga mandatos expresos y permita el ejercicio del derecho de acceso- es mucho más limitada que el concepto de transparencia en un sentido amplio, que es el que corresponde con la visión social y del periodismo sobre la transparencia.

En cualquier caso, todas estas previsiones nos llevan a preguntarnos por qué queremos la transparencia. La respuesta es que a la transparencia se le presumen diversas funciones para con el funcionamiento de la democracia. Villoria Mendieta (2014) resume muchas de sus virtudes como elemento necesario para la rendición de cuentas, la mejora la eficiencia de las organizaciones y la economía, la prevención de la corrupción, la configuración de un buen gobierno, e incluso el aumento de la calidad de la democracia.

Este papel instrumental lleva a considerar que el mayor acceso a la información permite a los ciudadanos ejercer sus derechos de forma más plena y realizar un escrutinio público que derive en una democracia de mayor calidad, ya que de lo contrario, "con poderes públicos sin ser sometidos a una efectiva rendición de cuentas, tanto institucional como ciudadana, es cuestión de tiempo que la calidad democrática languidezca y la desafección ciudadana crezca" (Hernández Ramos, 2018: 508). 
Periodismo, comunicación institucional y transparencia: aprendizajes de la crisis sanitaria del COVID-19

\subsection{Transparencia y periodismo: expectativas y realidad}

En el cumplimiento de estas funciones de la transparencia no se puede obviar el papel que desempeña el periodismo como uno de sus beneficiarios, y también porque ocupa un papel central como mediador entre la actividad gubernamental y la sociedad, ejerciendo un amplio protagonismo en el control democrático. Además, hay ventajas específicas y adicionales que ofrece la transparencia a la profesión periodística, desde la más básica, como es facilitar una mayor disposición de información, fuentes y datos. Es un complemento a la labor cotidiana de los periodistas porque abre la puerta a una mayor calidad y posibilidades de contrastar la información, haciendo posible el acceso a datos que "son oficiales, completos y certeros" (Díez Garrido y Campos Domínguez, 2018: 54). Permite "revelar la incoherencia del discurso político (más allá de la hemeroteca), a partir del análisis de los informes sobre acciones o medidas legislativas" (Magallón Rosa, 2013). Constituye una alternativa para obtener información que no pueden conseguir por otra vía o cuando interesa que sea información "cruda" o sin procesar (Bertoni, 2011). Ofrece una oportunidad para aumentar la autonomía ante la dependencia de las filtraciones interesadas o notas de prensa (Manfredi Sánchez, 2014: 78 y ss.; Magallón Rosa, 2013). También es frecuente señalar a la transparencia como medio de ruptura de las rutinas periodísticas permitiendo la creación de una agenda propia a partir de nuevas historias (Manfredi Sánchez, 2014: 77) y como punto de partida para el desarrollo de otras posibilidades de la profesión a través del periodismo de datos (Zafra Díaz, 2013).

Sin embargo, desde estas expectativas iniciales se ha transitado a considerar que su desarrollo a través de la Ley de Transparencia es insuficiente e impide aprovechar sus ventajas con plenitud (Díez Garrido y Campos Domínguez, 2018; Rubio Jiménez, 2017; La Rosa Barrolleta y Sandoval Martín, 2016). Según varios estudios a partir de encuestas a periodistas, la mayoría de los que conocían la Ley de Transparencia indicaba que sus medidas no resultaban bastantes para la labor periodística (Díez Garrido y Campos Domínguez, 2017) ${ }^{2}$; y que para dos de cada tres, no constituía una herramienta útil para el trabajo diario (Rubio Jiménez, 2017a: 37).

En el estudio de Díez Garrido y Campos Domínguez (2017) se mostraba que el $82,7 \%$ de los encuestados pensaba que era necesarias mejoras en la regulación del derecho de acceso a la información, y que los datos del portal de transparencia tampoco eran suficientes. Aparte, en este estudio se preguntaba por las funciones del periodismo al utilizar los mecanismos de transparencia, destacando prácticamente a partes iguales la difusión de información objetiva y la vigilancia al poder.

En el estudio de Rubio Jiménez (2017a: 37 y 38) de nuevo se hacía referencia en las encuestas a las limitaciones de la Ley de Transparencia y los encuestados resaltaban como causas principales la existencia de excepciones del acceso a la información, la

${ }^{2}$ Los porcentajes se distribuían entre un $56 \%$ que las consideraba insuficientes, un $24 \%$ que no conocía la normativa, un $10 \%$ que las consideraba suficientes y un $10 \%$ que ofrecía otras respuestas. 
Periodismo, comunicación institucional y transparencia: aprendizajes de la crisis sanitaria del COVID-19

carencia de un régimen sancionador del órgano de garantía -el Consejo de la Transparencia-y los tiempos de respuesta.

El estudio basado en entrevistas en profundidad de La Rosa Barrolleta y Sandoval Martín (2016) venía a confirmar parte de estas carencias. Con relación al derecho de acceso se aludía a los extensos tiempos de respuesta y a su incumplimiento reiterado; a la existencia de límites impuestos por la protección de datos, dando lugar a resoluciones negativas cuando se pide información en detalle sobre cargos públicos; o al hecho de que el silencio administrativo sea negativo (desestimatorio). Además, sobre el portal de transparencia se indicaba que su contenido no aporta información sustancialmente nueva, sino la repetida en otros lugares; mientras que respecto al Consejo de Transparencia y Buen Gobierno (CTBG) se apuntaba a la falta de un régimen sancionador directo para que el sistema funcionase eficazmente. Aparte, se manifiesta que todos estos motivos han contribuido a que no se haya podido desarrollar más aceleradamente un periodismo de datos en España manteniéndose "en un estado incipiente a pesar del boom que tuvo lugar a lo largo de 2015" (La Rosa Barrolleta y Sandoval Martín, 2016: 1224).

Las debilidades señaladas son, en su mayoría, una consecuencia de la configuración del derecho de acceso como un derecho de configuración legal de naturaleza administrativa, sin el rango y la especial protección que se otorga a los derechos fundamentales.

Como afirmaba Sánchez de Diego (2010: 256), el derecho de acceso es parte integrante del derecho de información contenido en el artículo 20 de la Constitución Española, "pues para informar y comunicar se necesita acceder a la información", tal y como contemplan textos básicos como la Declaración Universal de Derechos Humanos (art. 19) o el Pacto Internacional de Derechos Civiles y Políticos (art. 19.2), que especifican que la libertad de expresión e información contiene la posibilidad de investigar o buscar información. Sin embargo, esta desconexión mostrada a través de la LTBG fue de una de las principales críticas que recibió tras su publicación, porque de este modo "los ciudadanos y los periodistas no pueden acceder libremente a la información custodiada por el Gobierno y las administraciones" (Manfredi Sánchez, 2014: 73).

Al haber optado por esta vía en España —su no reconocimiento como un derecho fundamental-, una lógica consecuencia es que la regulación configure el derecho de una manera secundaria y tenga menor entidad a la hora de ponderar la colisión con otros derechos o intereses. La situación española va, por tanto, a contracorriente de los textos y sentencias de tribunales internacionales, cuyo panorama ha cambiado en las últimas décadas, dotando al derecho de acceso de un statu fundamental (Fernández Ramos, 2018: 233). De ellas, es destacable el criterio del Tribunal Europeo de Derechos Humanos (TEDH) que estima el carácter fundamental del derecho de acceso cuando está vinculado estrechamente a la libertad de expresión, de modo que se ha venido a configurar con un carácter instrumental (Hernández Ramos, 2018) Así en su Sentencia de 8 de noviembre de 2016 - caso Magyar-, venía a afirmar este 
Periodismo, comunicación institucional y transparencia: aprendizajes de la crisis sanitaria del COVID-19

reconocimiento cuando la información solicitada cumpla un test de cuatro requisitos: sea de interés público, sirva al debate público, esté disponible, y se solicitada por un guardián de la democracia (public watchdog), es decir, por un solicitante cualificado como son los periodistas. A tenor de esta interpretación, el derecho de acceso vendría a tener un carácter fundamental ante su ejercicio por un public watchdog y no por cualquier ciudadano, pero en cualquier caso ya supondría un avance respecto a la situación actual. Por ello, España, como país que ha suscrito el Convenio Europeo de Derechos Humanos y otras normas antes referidas, debería interiorizar este reconocimiento por la obligación constitucional (art. 10.2 CE) de interpretar los derechos fundamentales según los tratados y acuerdos internacionales suscritos por España (Cotino Hueso, 2017: 315).

\subsection{Información en época de crisis sanitaria}

Este reconocimiento hubiese tenido amplias consecuencias con relación al objeto tratado, ya que en el estado de alarma no se pueden suspender los derechos fundamentales —aunque sí condicionar su ejercicio- ${ }^{3}$, y el acceso a la información habría quedado resguardado ante su importancia en un contexto de crisis.

Hay que recordar que en estos contextos crece la demanda de información (Westlund y Ghersteti, 2015: 13), pero que se hace más importante aún porque cumple una triple función instrumental (De la Sierra Morón, 2020: 34 y 41): permite el control de la gestión gubernamental, ayuda a "la adopción responsable de decisiones individuales" de los ciudadanos, y contribuye a que otras administraciones públicas puedan actuar conforme a los riesgos definidos. En esta línea, se destaca el papel que desempeña como un elemento que favorece la confianza, porque sin ella "será muy difícil convencer a la gente de que adopte comportamientos necesarios de cara a tener ese riesgo bajo control" (Costa Sánchez y López García, 2020: 5). Pero conforme hay demanda de información, la información institucional y oficial constituye la fuente primordial para la elaboración de noticias, por ser el centro de atención y la referencia principal de consulta, aparte de que ante acontecimientos extraordinarios se incrementan las dificultades de los informadores privados (Ibáñez Peiró, 2020).

Por ello, en este marco aumenta la capacidad de comunicación de los gobiernos para marcar la agenda de los medios, de manera que la "repercusión mediática está directamente relacionada con las declaraciones de los responsables políticos" (Martínez Solana, 2004: 145). Se alteran así las condiciones que hacen que la comunicación

3 La Constitución Española no prevé la suspensión de los derechos fundamentales en el estado de alarma, pero sí establecer condicionamientos que vienen regulados en la Ley Orgánica 4/1981, de 1 de junio, de los estados de alarma, excepción y sitio. Entre ellos, están muchas de las limitaciones que se han producido por el entrecruzamiento de la situación de excepcionalidad y las medidas de prevención sanitaria, como el confinamiento o las limitaciones a la movilidad. La confluencia de ambos elementos estado de alarma y prevención de contagios- ha producido tales limitaciones, que parte de la doctrina considera un exceso porque se desdibuja el contenido esencial de algunos derechos fundamentales. Véase, por ejemplo, el texto de Arnaldo Alcubilla (2020) sobre el ejercicio del derecho de reunión en el estado de alarma.

Revista de Comunicación y Salud, 2020, Vol. 10, no 2, 569-591. 
política se defina como el resultado de una pauta de interacción constante entre políticos, comunicadores, periodistas y ciudadanos (Canel Crespo, 2006: 27). De hecho, en este contexto nos recuerda - salvando las distancias - la situación al equivalente de la comunicación política de posguerra descrita por Lasswell en 1948 en un contexto de propaganda gubernamental, comunicación lineal y unidireccional, que desde una posición de superioridad se dirige a una masa pasiva sobre la que se podía influir.

Además, no se puede obviar que las declaraciones gubernamentales forman parte del puzzle de la comunicación institucional, cuyas técnicas tienen una intención persuasiva para influir en los destinatarios y lograr su adhesión (Canel Crespo, 2007). Por ello no es ajena a la utilización de posibles recursos como la cesión de información, el ocultamiento, la escenificación y la comunicación persuasiva.

De ahí, que la transparencia aumente su importancia, porque permita la disposición de fuentes de información alternativa y de datos que no estén acompañados de la visión gubernamental, de manera que sean los periodistas los que puedan construir su propia agenda y contrastar adecuadamente las declaraciones e información gubernamental (Manfredi Sánchez, 2014; Magallón Rosa, 2013).

Estos planteamientos confluyen de nuevo en la idea de que la transparencia y su utilización por los profesionales del periodismo son un contrapeso ante las tácticas de comunicación de los gobiernos, más aún en épocas como los estados excepcionales, en los que se produce un relajamiento de los controles habituales -0 una mayor dificultad para su desarrollo ordinario-, junto a una mayor concentración de poder, sobre la que, además, existe un aumento de la predisposición ciudadana a tolerar que se vea afectado el ejercicio de libertades (Amat et al., 2020), con los riesgos de involución democrática que ello conlleva ${ }^{4}$.

\section{ANÁLISIS DE CASO: INFORMACIÓN Y TRANSPARENCIA DURANTE EL ESTADO DE ALARMA}

Una vez expuesta la bibliografía principal que ofrece un marco sobre la transparencia en época de crisis, es necesario introducir una descripción sobre el interés informativo en el contexto de pandemia y las actuaciones de comunicación gubernamental, para pasar posteriormente al análisis de las causas por las que se achacaba la falta de transparencia con relación al derecho de acceso y la publicidad activa.

\footnotetext{
${ }^{4}$ Según el estudio de Amat, Falcó, Arenas y Muñoz (2020) sobre la actitud de los ciudadanos ante la crisis del COVID, los resultados de encuesta muestran que los ciudadanos están más predispuestos a asumir la concentración temporal de poder gubernamental aun a costa de la restricción de libertades.
} 
Periodismo, comunicación institucional y transparencia: aprendizajes de la crisis sanitaria del COVID-19

\subsection{Interés informativo y comunicación gubernamental}

Desde los días previos a la declaración del estado de alarma se incrementó ostensiblemente el consumo de medios informativos por parte de la ciudadanía, ya no solo por la expectación ante los acontecimientos, sino por la situación de contexto en la que los ciudadanos habían quedado confinados en sus hogares, con condiciones más favorables para el acceso al consumo de información sin los constreñimientos del ritmo ordinario de la actividad cotidiana.

Tomando como indicador los datos de audiencia de televisión ofrecidos por Barlovento Comunicación, desde una situación más o menos estable de consumo de televisión en enero y febrero de 2020 de menos de $4 \mathrm{~h}$. diarias por persona —con datos inferiores al mismo periodo del año anterior-; se pasó a una situación de consumo en torno a las $4,5 \mathrm{~h}$. en marzo y $5 \mathrm{~h}$. en abril, con un incremento en torno a una hora diaria respecto a los mismos datos del ejercicio anterior; mientras que en los meses de mayo y junio se empezaron a moderar estos incrementos (Tabla 1).

Tabla 1. Evolución del tiempo de consumo de televisión. Enero-mayo de 2020

\begin{tabular}{|l|c|c|c|c|c|c|}
\hline \multicolumn{1}{|c|}{ Televisión } & $\begin{array}{c}\text { Enero } \\
2020\end{array}$ & $\begin{array}{c}\text { Febrero } \\
2020\end{array}$ & $\begin{array}{c}\text { Marzo } \\
2020\end{array}$ & $\begin{array}{c}\text { Abril } \\
2020\end{array}$ & $\begin{array}{c}\text { Mayo } \\
2020\end{array}$ & $\begin{array}{c}\text { Junio } \\
2020\end{array}$ \\
\hline $\begin{array}{l}\text { Tiempo diario por } \\
\text { persona }\end{array}$ & $3: 57$ & $3: 44$ & 4,44 & $5: 02$ & $4: 20$ & $3: 45$ \\
\hline $\begin{array}{l}\text { Incremento respecto al } \\
\text { mes del año anterior }\end{array}$ & $-10 \mathrm{~min}$. & $-16 \mathrm{~min}$. & $+51^{*} \mathrm{~min}$. & $+69 \mathrm{~min}$. & $+37 \mathrm{~min}$. & $+10 \mathrm{~min}$. \\
\hline
\end{tabular}

Fuente: análisis mensual del comportamiento de la audiencia TV. Barlovento Comunicación (www.barloventocomunicacion.es).

En este marco, la demanda informativa también aumentó, en la línea que se produce ante situaciones de crisis (Westlund y Ghersteti, 2015: 13). Los datos del barómetro del CIS del mes de marzo ${ }^{5}$ reflejaban que el $71,2 \%$ de los ciudadanos seguía la información con mucho o bastante interés, un dato muy superior al nivel de interés de eventos como la campaña electoral de los comicios generales de noviembre de $2019^{6}$. Aparte, un $54,5 \%$ afirmaba en ese periodo inicial de la crisis, que le gustaría tener más información sobre las medidas de prevención. En posteriores barómetros del CIS (abril - junio) no hubo preguntas equivalentes, salvo la relativa a la suficiencia de información gubernamental, que en el mes de abril mostraba que más de un $60 \%$ demandaba más información, en general $(58,2 \%)$ o más veraz $(2,4 \%)$.

Por tanto, la demanda de información a lo largo de los meses de marzo y abril de 2020 justificaba, aún más, la importancia de la actividad periodística respecto a la

${ }^{5}$ El barómetro CIS núm. 3277 de marzo de 2020 se realizó justo antes de la declaración del estado de alarma entre el 1 y el 13 de marzo.

${ }^{6}$ Según el barómetro de diciembre de 2019 (estudio CIS núm. 3269), el 35,6\% de los encuestados siguió la campaña electoral con mucho o bastante interés. 
Periodismo, comunicación institucional y transparencia: aprendizajes de la crisis sanitaria del COVID-19

producción de noticias en número y calidad suficiente, así como la puesta a su disposición de todos los recursos posibles para el desarrollo de su trabajo.

A lo largo del periodo se pusieron en marcha ruedas de prensa diarias, se confeccionaron dosieres sobre la evolución de la enfermedad -emanados del Ministerio de Sanidad-, y se ofreció información constante a través de las páginas web de los ministerios ${ }^{7}$, entre otras muchas actuaciones de comunicación ${ }^{8}$. No obstante, todas ellas tenían como común denominador la unidireccionalidad de los mensajes y la preponderancia de las versiones oficiales sobre los acontecimientos.

Así, el primer hito en la controversia sobre la falta de transparencia fueron las quejas de los periodistas y de los medios de comunicación por las ruedas de prensa con preguntas elegidas por el gobierno. Esta situación se alargó durante las primeras semanas del estado de alarma. Primero sin la presencia de periodistas, quienes remitían sus preguntas previamente, siendo seleccionadas por la Secretaría de Estado de Comunicación; y posteriormente, ya mediante videoconferencia con intervención de los periodistas, pero sujetas de igual modo a la selección gubernamental de los medios que las podían formular.

Este modo de proceder se ha criticado por algunos autores por el hecho de que la selección de preguntas supone un mecanismo de censura directa (Espinosa Sánchez, 2020), aparte de que el primer sistema en diferido - que no permitía la réplica o repreguntar- era de difícil justificación ante las posibilidades que ofrecen las nuevas tecnologías. En cualquier caso, el efecto de este sistema es que se ofreciese "una imagen de la gestión del Gobierno que no se correspondía con la realidad esquivando los temas más espinosos, para no poner en tela de juicio la actuación gubernamental" (Ibáñez Peiró, 2020: 313).

Con todo, esta situación llevó al manifiesto "libertad para preguntar" (01/04/2020) , en un contexto en el que aumentaban las críticas. Éstas se fundaban en las dudas

7 La información que integra la comunicación gubernamental se fue volcando en las páginas web de La Moncloa (https://www.lamoncloa.gob.es/) y del Ministerio de la Presidencia (https://www.mpr.gob.es/). No obstante, Ministerio de Sanidad fue incluyendo información diaria sobre la evolución de la pandemia, al igual que se incorporaron contenidos en el Punto de Acceso General, el Portal de Transparencia y prácticamente en todos los Ministerios respecto a su ámbito de actuación.

8 Ibáñez Peiró (2020) cuantifica en 118 las ruedas de prensa relacionadas con el Coronavirus entre el 10 de marzo y el 30 de abril.

${ }^{9}$ El manifiesto fue suscrito por cientos de periodistas de diversas tendencias editoriales y venía a criticar el sistema de selección de preguntas y a demandar las ruedas de prensa telemáticas mediante videollamadas. Entre los pasajes del manifiesto se hacían criticas duras al Gobierno que se sintetizan en el siguiente párrafo: "Estas líneas no tienen como objetivo defender ni condenar la gestión del Gobierno en la crisis del coronavirus, sino garantizar que los medios de comunicación puedan realizar su función sin mordazas y sin dificultades añadidas. Estas no son más que excusas para controlar a la prensa. Que las preguntas las formule un miembro del propio Gobierno revela falta de transparencia y un interés por controlar la información. Su consecuencia deriva en una nueva forma de censura a los medios y un desprecio intolerable a los mismos ciudadanos a los que se reclama enormes esfuerzos que mayoritariamente están cumpliendo a rajatabla". 
Periodismo, comunicación institucional y transparencia: aprendizajes de la crisis sanitaria del COVID-19

sobre la calidad de los datos aportados por el gobierno respecto a la evolución de los contagios y por la falta de contraste con las cifras de mortalidad que ofrecían otras fuentes de información (Costa Sánchez y López García, 2020: 6 y 7) 10 $^{10}$.

Estas reacciones provocaron que el Gobierno propusiera un nuevo sistema de videoconferencias a principios de abril. Sin embargo, de nuevo se propagó la indignación del periodismo y de los medios de comunicación tras la publicación, a mediados de abril del Barómetro del CIS, que incluía entre sus preguntas la medición del grado de acuerdo con la posibilidad de prohibir la difusión de información que no estuviese alineada con las fuentes oficiales. La inserción de esta pregunta fue interpretada como una invitación a la censura estatal y se acusaba al gobierno de buscar una respuesta favorable mediante una redacción de la pregunta alejada de la neutralidad ${ }^{11}$.

Con posterioridad, surgieron otras polémicas al detectar que se había paralizado la resolución de solicitudes de información pública y que no se estaba publicando la información de los contratos. De ahí, que empezase a surgir una espiral de reacción algo tardía - de las organizaciones relacionadas con la transparencia y el derecho de acceso, dando a conocer diversos manifiestos y comunicados por la Coalición Pro Acceso, CIVIO, Transparencia Internacional, Access INFO, o la Asociación de Profesionales de la Transparencia (ACREDITRA), entre otros.

A todas estas situaciones se añadieron nuevas circunstancias derivadas de las demandas de información de periodistas y organizaciones, al observar que no se publicaba, ni se facilitaba la composición de los miembros del Comité de Expertos que asesoraba al gobierno o los informes de desescalada, información que era de carácter público según la Ley General de Salud Pública (Ley 33/2011, de 4 de octubre).

Todo ello, llevó a que se hablase de falta de transparencia y censura, y que los medios de comunicación incorporasen a la agenda mediática noticias relacionadas con el portal de transparencia y la libertad de información.

La principal reacción del gobierno ante las primeras críticas fue la argumentación de que se estaba ofreciendo más información que nunca, enfatizando en la constante actividad de comunicación institucional que se desarrollaba. Aparte, se produjeron algunas rectificaciones, permitiendo preguntas por videoconferencia o publicando las adjudicaciones de contratos, pero sin reactivar los plazos del derecho de acceso (se

10 Otra situación que se añadía con relación a los medios de comunicación fue la aprobación de ayudas para el mantenimiento de sectores esenciales, que al incorporar entre ellos a grandes grupos de medios de comunicación, se interpretó como un intento del gobierno para su utilización estratégica con la finalidad de favorecer la imagen sobre su actuación (Espinosa Sánchez, 2020).

${ }^{11}$ Así, por ejemplo, el diario El Mundo (15/04/2020) ilustraba una de sus noticias con el siguiente titular: "Tezanos fabrica un CIS para reclamar apoyo a Pedro Sánchez y plantear la censura a los medios".

Revista de Comunicación y Salud, 2020, Vol. 10, oㅡ 2, 569-591. 
Periodismo, comunicación institucional y transparencia: aprendizajes de la crisis sanitaria del COVID-19

reanudaron el 1 de junio de 2020) y sin facilitar los nombres e informes del comités de expertos que le asesoraba y que existía según el Gobierno ${ }^{12}$.

\subsection{La problemática del derecho del acceso}

Como se ha mencionado, se había empezado a detectar en foros de expertos las consecuencias de la interrupción de plazos administrativos del Decreto del estado de alarma con relación al ejercicio del derecho de acceso a la información pública. Esta suspensión tenía como consecuencia la dilatación indefinida del tiempo para responder a las solicitudes de transparencia. El blog del experto Miguel Ángel Blanes Climent (2020), y sobre todo, su entrada titulada El derecho de acceso sobre el Coronavirus durante el estado de alarma (31 de marzo de 2020), tuvo una amplia repercusión en el ecosistema de organizaciones de la transparencia, que fue seguida por otros artículos de amplio impacto como el blog de la Fundación Hay Derecho (Ibáñez García, 2020).

En efecto, la publicación del Real Decreto, núm. 463/2020, de 14 de marzo, por el que se declara el estado de alarma para la gestión de la situación de crisis sanitaria ocasionada por el COVID-19, venía a suspender los plazos administrativos con un alcance a todo el sector público. El ejercicio del derecho de acceso sigue procedimientos sujetos al derecho administrativo y, por tanto, su paralización incide en el tiempo del que disponen las administraciones públicas para resolver las solicitudes.

Según la Ley de Transparencia, las solicitudes de acceso a la información pública deben ser resueltas en el plazo ordinario de un mes desde que son recibidas por el órgano competente (art. 20.1 LTBG). Tal y como está formulada la regulación, este plazo puede demorarse en el lapso de tiempo que transcurre desde que se formula la solicitud, hasta que la recibe el órgano competente. Aparte, es susceptible de su ampliación un mes más si la información solicitada es voluminosa o compleja.

Por ello, la interrupción de plazos afectaba con carácter general al derecho de acceso. La redacción original de la disposición adicional tercera (apdo. 4) del RD de declaración del estado de alarma, contenía una previsión para que la suspensión de plazos no afectase a aquellos procedimientos y resoluciones "cuando estos vengan referidos a situaciones estrechamente vinculadas a los hechos justificativos del estado de alarma". Es decir, se vendrían a salvaguardar aquellos procedimientos que respondiesen a la rendición de cuentas sobre el estado de alarma, entre los que podía incardinarse las solicitudes de información pública. Sin embargo, cuatro días más tarde se venía a modificar este apartado ${ }^{13}$, pasando a ser una posibilidad que se podría

\footnotetext{
12 Los informes de desescalada se fueron publicando en la página web del Ministerio de Sanidad, una vez que todas las Comunidades Autónomas habían ido transitando hacia el desconfinamiento. Sin embargo, la identidad de los miembros del Comité de Expertos no se publicó, y a fecha de cierre de este artículo (30/07/2020), saltó de nuevo la polémica al darse a conocer que en comunicaciones oficiales del Ministerio de Sanidad al Defensor del Pueblo se negaba que hubiese existido un Comité de Expertos.

13 Real Decreto 465/2020, de 17 de marzo, por el que se modifica el Real Decreto 463/2020, de 14 de marzo, por el que se declara el estado de alarma para la gestión de la situación de crisis sanitaria
} 
Periodismo, comunicación institucional y transparencia: aprendizajes de la crisis sanitaria del COVID-19

"acordar motivadamente", es decir, que imponía un requisito adicional para que se continuasen los plazos - la motivación-, en lugar de la redacción anterior que imponía de manera generalizada su continuación si tenían relación con la justificación del estado de alarma.

De este modo, cualquier solicitud de datos o información relacionada con la crisis del COVID-19 se veía paralizada, aunque existiese una posibilidad a discrecionalidad de la administración para acordar la continuación de plazos. Además, la importancia esta suspensión era de amplio alcance, porque no se limitaba al ámbito del Estado, ya que las normas de procedimiento administrativo son de aplicación al resto del sector público, y por tanto, tenía alcance general a todas las administraciones públicas de nuestro país ${ }^{14}$.

De ahí que fuese objeto de crítica porque suponía una paralización del derecho de acceso y porque, además, no se observaba justificación alguna para estas previsiones porque los empleados públicos seguían desempeñando su labor a través del teletrabajo y porque su mantenimiento no era perjudicial para la situación sanitaria. Como bien ha indicado De la Sierra Morón (2020), pese a que la LTBG establece una serie de límites para el derecho de acceso, "no parece que en este caso resulten de aplicación ante el bien jurídico superior que constituye la salud pública". Además, la continuación de plazos se podría haber exceptuado de cualquier paralización, tal y como se hizo con otras materias como los plazos tributarios y de la seguridad social. No obstante, la suspensión fue comunicada al CTBG sin que se manifestase al respecto (Nota de la Dirección General de Gobernanza Pública de 17 de abril de 2020).

El impacto en la paralización de las resoluciones se puede observar claramente a partir de los datos de las estadísticas del Portal de Transparencia del Gobierno de España. Entre los meses de marzo a mayo de 2020, el dimensionamiento del atasco en las solicitudes de acceso en tramitación creció ostensiblemente. En las condiciones ordinarias del mes de febrero se situaba en las 893 solicitudes pendientes, que prácticamente se duplicaron al finalizar el mes abril llegando hasta las 1.612 solicitudes, para aumentar de nuevo hasta las 1.997 en mayo de 2020 (TABLA 2). Aparte, los datos acumulados de las solicitudes hasta febrero de 2020, mostraban que las dirigidas a sanidad participaban en torno al 3,9\% del total, pero este porcentaje creció entre las solicitudes nuevas formuladas durante el estado de alarma hasta el 6,6\% en marzo, $17,6 \%$ en abril y $18,6 \%$ en mayo, convirtiéndose en la principal materia sobre la que versaban las solicitudes de acceso.

ocasionada por el COVID-19. BOE núm. 73/2020, de 18 de marzo. Disponible en: https://www.boe.es/boe/dias/2020/03/18/pdfs/BOE-A-2020-3828.pdf

14 No es objeto de este artículo describir la situación en las Comunidades Autónomas y el resto de administraciones públicas, pero cabe mencionar que la situación fue asimétrica entre ellas. Así, algunas Comunidades Autónomas decidieron continuar la resolución de solicitudes de acceso con normalidad, mientras que otras resolvían según la materia, o bien, dejaron sin continuar las tramitaciones hasta la reanudación de los plazos administrativos.

Revista de Comunicación y Salud, 2020, Vol. 10, no 2, 569-591. 
Periodismo, comunicación institucional y transparencia: aprendizajes de la crisis sanitaria del COVID-19

Una observación adicional es que el número de nuevas solicitudes se vio reducido en el contexto de crisis (de 966 en febrero a la cifra de 585 en abril), por lo que la envergadura de la falta de tramitación podría haber sido mucho mayor si hubiese continuado el ritmo ordinario de solicitudes de información.

Tabla 2. Estado de las solicitudes de acceso a la información durante el estado de alarma

\begin{tabular}{|l|r|r|r|}
\hline \multicolumn{1}{|c|}{ Solicitudes de acceso } & \multicolumn{1}{c|}{ Marzo } & \multicolumn{1}{c|}{ Abril } & \multicolumn{1}{c|}{ Mayo } \\
\hline Nuevas & 727 & 585 & 618 \\
\hline \% solicitudes nuevas dirigidas a sanidad & 6,6 & 17,6 & 18,6 \\
\hline En tramitación & 1.080 & 1.612 & 1.997 \\
\hline
\end{tabular}

Fuente: elaboración propia a partir del informe de estadísticas del Portal de Transparencia del Gobierno de España.

\subsection{Publicidad activa: contratos públicos, comités de expertos e información sanitaria.}

Sobre el otro pilar principal de la transparencia -la publicidad activa - hay que mencionar que la LTBG regula las materias que deben publicar obligatoriamente todas las entidades que están en su ámbito de aplicación. Sin embargo, en lo que respecta a la crisis del COVID, las principales controversias se suscitaron con relación a los mandatos de publicación o conocimiento público que derivaban de otras leyes en materia de contratación y salud pública.

En el seno del Observatorio de la Contratación Pública ${ }^{15}$ ya se estaba llamando la atención sobre los contratos de emergencia, que por sus características prescindían de los elementos de publicidad habituales y que, además, no se estaban publicando las adjudicaciones inmediatamente (Gamero Casado, 2020).

El origen de la situación hay que buscarlo días antes de la declaración del estado de alarma. El Real Decreto Ley 7/2020, de 12 de marzo, habilitaba la contratación por el procedimiento de emergencia para atender las necesidades derivadas del COVID-19 (art. 16). Este procedimiento previsto en la Ley de Contratos del Sector Público (art. 120) tiene un amplio recorrido en la legislación española y permite contratar sin atender ningún tipo de procedimiento, incluso habilitando la contratación verbal (García Melián, 2020).

Las consecuencias prácticas en lo relativo a la publicidad de los contratos es que no hay anuncios previos de las licitaciones, aunque no obsta para publicar la formalización de los contratos, que debe llevarse a cabo en el plazo de 15 días en el perfil del contratante y en el BOE para los contratos de la Administración General del Estado y

15 El Observatorio de Contratación Pública es un foro de debate y análisis de expertos y profesionales sobre la materia, cuyas publicaciones se pueden encontrar en la dirección: http://www.obcp.es/

Revista de Comunicación y Salud, 2020, Vol. 10, oㅡ 2, 569-591. 
entidades vinculadas (art. 154 LCSP). Además, la contratación pública es objeto de una obligación expresa de publicidad activa según la Ley de Transparencia. Por tanto, al no existir ninguna excepción en la Ley de Contratos y en la Ley de Transparencia, deben cumplir con las obligaciones de publicidad, porque en su defecto se impide el adecuado control del respeto a las normas y principios básicos de la contratación pública (Gamero Casado, 2020).

Así, se detectó que faltaba información sobre estos contratos en el Portal de Transparencia y en la Plataforma de Contratación del Estado, los cuáles no se comenzaron a publicar hasta pasado más de un mes desde la declaración del estado de alarma.

En cualquier caso, la polémica suscitada sobre la falta de publicidad de los contratos públicos, no solo se produjo por la inobservancia respecto a las contrataciones no publicadas en plazo, sino porque además, se había dejado sin efecto una forma de solicitarlas como es el derecho de acceso. Aparte, la utilización de este trámite de emergencia es más opaco y dispone de menos controles, alejándose del espíritu y de la práctica que debe guiar la contratación pública.

Finalmente, se hace alusión a otras obligaciones de transparencia contenidas en la Ley General de Salud Pública de 2011, como es la identidad de los miembros del Comité de Expertos que asesoraba al Gobierno respecto al Covid-19 y a sus informes, entre los que estaban las evaluaciones de las Comunidades Autónomas para la desescalada.

Esta información fue demandada sin éxito por los periodistas y también por algunas Comunidades Autónomas. Al respecto, la empresa de fact-checking Maldita, denunció esta situación al CTBG, a cuya solicitud respondía mostrando uno de los problemas de la insuficiencia de la Ley de Transparencia. El Consejo de Transparencia venía a indicar que la composición de estos comités de expertos no formalizados, no son el tipo de órganos a los que se refiere la LTBG, y que por tanto, el CTBG no disponía de competencias para realizar un control de la publicidad activa exigida por otras normas distintas a la Ley de Transparencia.

Por otra parte, la Ley General de Salud Pública no dispone de un mecanismo para exigir la publicación de las obligaciones que establece. Transcurrida prácticamente una década desde su aprobación, no existe un reglamento de la ley, ni un procedimiento para reclamar la difusión de información, lo que se traduce en una obligación sin ningún tipo de garantías de cumplimiento. 
Periodismo, comunicación institucional y transparencia: aprendizajes de la crisis sanitaria del COVID-19

\section{DISCUSIÓN Y CONCLUSIONES}

La situación originada tras la declaración del estado de alarma, combinada con la paralización de la actividad económica, el confinamiento en los hogares y la posición del Gobierno como fuente prácticamente exclusiva de información, provocaba una amplia concentración de poder en torno al ejecutivo. Por ese motivo, la transparencia reafirma su importancia para ejercitar control público y favorecer la rendición de cuentas, sin que quepa considerar que la transparencia es sustituida por la comunicación institucional, porque el objetivo y protagonista de cada una son distintos. Mientras que la comunicación institucional busca en última instancia la adhesión (Canel Crespo, 2007) —en este caso en torno al gobierno-; la transparencia tiene como objetivo el escrutinio y el acceso a lo público, siendo la ciudanía su protagonista principal. También difiere en que la transparencia es neutra -es información y datos-, por lo que se aleja de las connotaciones y los marcos de interpretación que se utilizan en comunicación. Aparte, la comunicación queda a arbitrio del emisor, mientras que la transparencia se centra en los hechos y supone un derecho que no debería quedar a voluntad del que posee la información.

Aparte de esta disquisición, se ha observado desde el primer momento como la regulación de obligado cumplimiento en nuestro país no garantiza la transparencia, ni la rendición de cuentas. Por si sola, la Ley de Transparencia se muestra insuficiente, pero no solo eso, sino que además se comprueba su fragilidad en el entramado jurídico, que permite un vaciamiento de sus obligaciones ante la interrupción de plazos administrativos y que exista una la falta de efectividad ante trámites u obligaciones contenidas en otras normas.

Una de las principales causas por las que el derecho de acceso está a merced de las normas administrativas es que no está reconocido como un derecho fundamental (Sánchez de Diego y Sierra Rodríguez, 2020; Cotino Hueso, 2017). Este es uno de los principales retos a solventar para que tenga una mayor efectividad, bien a través de una nueva ley de transparencia que conlleve este reconocimiento, o a partir de la labor de jueces y tribunales que terminen construyendo una interpretación del derecho de acceso como derecho fundamental.

Al margen del debate respecto a que sea o no predicable un derecho fundamental de acceso para todos los ciudadanos, como mínimo, debía serlo para el colectivo de periodistas, en la medida en que es necesario el acceso a la información pública para un despliegue efectivo de la libertad de información y porque actúan como public watchdogs (Sánchez de Diego, 2010; Cotino Hueso, 2017). En cualquier caso, su reconocimiento como derecho fundamental podría llevar a una mayor fortaleza del derecho de acceso ante la interpretación del alcance de sus límites, y podría minorar la barrera que supone para la profesión periodística muchas de las limitaciones derivadas de la protección de datos de carácter personal y que impiden acceder a información sobre los cargos públicos (La Rosa Barrolleta y Sandoval Martín, 2016).

Revista de Comunicación y Salud, 2020, Vol. 10, no 2, 569-591. 
Periodismo, comunicación institucional y transparencia: aprendizajes de la crisis sanitaria del COVID-19

Mientras llega el momento de este reconocimiento, hay una serie de cambios legales en la normativa de transparencia que se han evidenciado como necesarios en el contexto de pandemia. El ejercicio del derecho de acceso, tal y como está configurado no es útil para el periodismo por los tiempos de respuesta, siendo una de las principales críticas mostradas en los estudios analizados (Díez Garrido y Campos Domínguez, 2017; Rubio Jiménez, 2017; La Rosa Barrolleta y Sandoval Martín, 2016). Es cierto que estas solicitudes se pueden realizar $y$, mientras tanto, ir completando las investigaciones a la espera de respuesta (Magallón Rosa, 2013), pero el plazo de treinta días ante situaciones de vertiginosa rapidez es excesivo. Aun cuando no se hubiese paralizado el plazo para responder a las solicitudes de acceso, tal y como está previsto, ha quedado patente que la regulación de su ejercicio no se acomoda a las necesidades del periodismo.

Por ello, se rescatan algunas propuestas como la formulada por Rubio Jiménez (2017b: 456 y ss.), quien plantea un sistema de dos velocidades, una ordinaria, que podría corresponder con la configuración de los treinta días actuales; y una extraordinaria, que en pocos días obligase a resolver. No obstante, esta autora no cree que deba establecerse un cauce privilegiado para los periodistas, sino que sea una opción para cualquier persona, aunque la indudable conexión de la labor del periodismo con la libertad de información debería tener algún tipo de garantías adicionales.

Aun así, una resolución acelerada de las peticiones tampoco asegura que no sean inadmitidas o rechazadas. Por ello, se hace necesaria una reflexión y la mejora de la regulación para que la interpretación de límites y causas de inadmisión sea aplicada de manera restrictiva.

Aparte, estos cambios se deberían acompañar de un procedimiento acelerado de reclamación ante el Consejo de la Transparencia y Buen Gobierno. Sería un contrasentido que las solicitudes aceleradas tuviesen una respuesta negativa o falta de respuesta, y que las reclamaciones ante el órgano de garantía del derecho, llevasen a una amplia dilación de tiempos. Con relación a este planteamiento, de nada sirve unos procedimientos ágiles de reclamación si posteriormente el CTBG no tiene fuerza para obligar al cumplimiento de sus decisiones. Al respecto, debería acoger competencias sancionadoras directas — debilidad detectada por el periodismo (Rubio Jiménez, 2017a: 37 y 38; La Rosa Barrolleta y Sandoval Martín, 2016) -, pero también fortalecer sus resoluciones otorgándoles fuerza vinculante y ejecutiva. De este modo, ante una resolución favorable al acceso, la resolución podría ser título bastante como para exigir la entrega de la información ante cualquier funcionario o autoridad pública. Hoy en día sin embargo, ni tiene este valor, ni asegura la entrega de la información, y además, una entidad pública puede demorar sucesivamente la entrega de la información recurriendo las resoluciones del CTBG ante la jurisdicción contencioso-administrativa.

Con relación al caso estudiado, hemos visto que el mandato informativo de la Ley General de Salud Pública queda desprovisto de cualquier efectividad si no recoge un mecanismo para asegurar su cumplimiento, y que el CTBG no dispone de competencias para actuar fuera de las obligaciones de la LTBG. De ahí se deriva la

Revista de Comunicación y Salud, 2020, Vol. 10, oㅡ 2, 569-591. 
Periodismo, comunicación institucional y transparencia: aprendizajes de la crisis sanitaria del COVID-19

necesidad de una coordinación de la Ley de Transparencia con el resto de obligaciones de información dispuestas por otras leyes sectoriales o regímenes especiales (Fernández Ramos, 2018: 238). En esta línea, se debería habilitar el Consejo de la Transparencia y Buen Gobierno para que pueda actuar ante cualquier incumplimiento de obligaciones de publicidad e información fuera del ámbito de la LTBG.

En el caso concreto de la contratación de emergencia y sin poner en duda la conveniencia de su régimen de ante situaciones extraordinarias, su regulación debe compatibilizarse con las posibilidades que hoy en día permiten las nuevas tecnologías para la publicación de información. Nos encontramos de nuevo con un acortamiento de los plazos como propuesta principal, poniendo al mismo nivel la agilidad que se permite para contratar con su publicación. La inmediatez a la hora de publicar los contratos podría ser la pauta a seguir, sin perjuicio de que aquella información que requiera tareas de elaboración —como los informes de justificación- se puedan incluir posteriormente.

Por otra parte, se ha observado que a lo largo de esta crisis ha existido una polémica constante sobre la calidad de la información respecto a los afectados y los ingresados en las UCls. La transparencia de los datos sanitarios es una tarea pendiente que exige un reforzamiento de las obligaciones de publicidad. Una alternativa hubiese sido la elaboración de información propia a partir de datos en bruto, pero como ya se ha advertido sobre el periodismo de datos (La Rosa Barrolleta y Sandoval Martín, 2016), las previsiones de la regulación en la LTBG y en el resto de legislación no contribuyen a su desarrollo. La práctica durante esta crisis ha sido ofrecer la información sanitaria a través de datos agregados en documentos PDF, lo que a su vez ha destapado los problemas de aplicación de metodologías distintas y con diferente periodicidad en las Comunidades Autónomas, por lo que se hace más lejana cualquier expectativa sobre el acceso a datos en bruto debido a la asimetría de cultura de datos abiertos que existe en el ámbito autonómico (Curto Rodríguez, 2020) Por otra parte, cuando se han publicado los registros individuales de contratos de emergencia, las posibilidades de descarga sencilla en formato de hoja de cálculo a través del Portal de Transparencia son limitadas. Esto origina que se deba hacer un proceso manual de filtrado y extracción que hace tediosa cualquier explotación propia, pero que, en cualquier caso, refleja la falta de previsiones para que la información sobre contratación pública sea fácilmente explotable por terceros (Beltrán Orenes y Martínez Pastor, 2016).

De todas las circunstancias descritas se desprende que habiendo transcurrido algunos años desde la puesta en marcha de la Ley de Transparencia y del despliegue de las estructuras de la transparencia -en el Estado y en las Comunidades Autónomas-, éstas no responden a las expectativas y funciones que se suponen a la transparencia.

En situaciones de crisis como esta, en la que además de la mencionada concentración de poder se produce un relajamiento de los controles habituales, la importancia de la transparencia es mayor aun ante el riesgo de abuso de poder y la mayor tolerancia a la restricción de libertades (Amat et al., 2020). Por ello, no carecería

Revista de Comunicación y Salud, 2020, Vol. 10, no 2, 569-591. 
Periodismo, comunicación institucional y transparencia: aprendizajes de la crisis sanitaria del COVID-19

de lógica pretender que se incorporen obligaciones de transparencia y de creación de portales informativos específicos ante la declaración de cualquiera de los estados excepcionales que se puedan producir por crisis sanitarias o equivalentes. No obstante, no estaríamos hablando con la misma intensidad de esta problemática si hubiese una cultura de la transparencia y rendición de cuentas arraigada en nuestro país, sobre todo, entre las personas que ocupan posiciones de gobierno, de manera que sin la necesidad de una ley tomasen una actitud activa para ofrecer toda la información y no pretender su sustitución por la comunicación institucional.

Por todo ello, hay que reiterar que es necesario reeditar la regulación de la transparencia, con un alcance mucho mayor, con un reconocimiento del acceso a la información pública como derecho fundamental, y con una proyección transversal para evitar que se mantengan ámbitos de opacidad que escapen del control de los órganos de garantía a los que hay que dotar de una mayor fortaleza. Como idea final de este artículo, se sintetizan todas las insuficiencias que muestra la transparencia para con la labor periodística en la carencia de un estatuto privilegiado de acceso a la información pública que esté adaptado a sus necesidades mediante un acceso ágil y completo a la información que obra en manos de las instituciones públicas.

\section{REFERENCIAS}

Amat, F; Arenas, A.; Falcó Gimeno, A.; y Muñoz, J. (2020). Pandemics meet democracy: Experimental evidence from the COVID-19 crisis in Spain. SocArXiv (working paper). Recuperado de https://arxiv.org/abs/2003.03848

Arnaldo Alcubilla, E. (2020). "Salus populi est lex, sed lex superior est constitutio". Diario La Ley, núm. 9637, Sección Tribuna.

Barredo Rodríguez, C. (2017). Las causas de inadmisión de las solicitudes de acceso a la información; en particular, la doctrina de las autoridades independientes de transparencia. Revista General de Derecho Administrativo, 46. Recuperado de http://laadministracionaldia.inap.es/noticia.asp?id=1507961

Beltrán Orenes, P. y Martinez Pastor, E. (2016). Grado de cumplimiento de las Leyes de transparencia, acceso y buen gobierno, y de reutilización de los datos de contratación de la Administración central española. El profesional de la información, 25 (4), 557-567. doi: 10.3145/epi.2016.jul.05

Bertoni, E.A. (2011). Libertad de Información ¿Tres palabras inofensivas? Leyes de acceso a la información y rol de la prensa. Washington: Banco Mundial. Recuperado de https://www.palermo.edu/cele/pdf/Bertoni-LibertaddeInformacionyPrensa.pdf

Blanes Climent, M.A. (2020). "El derecho de acceso sobre el Coronavirus durante el Estado de Alarma" [entrada de blog] https://miguelangelblanes.com/2020/03/ 
Periodismo, comunicación institucional y transparencia: aprendizajes de la crisis sanitaria del COVID-19

Canel Crespo, M.J. (2007). Comunicación de las instituciones públicas. Madrid: Editorial Tecnos.

Canel Crespo, M.J. (2006). Comunicación política. Una guía para su estudio y práctica. Madrid: Tecnos.

Costa Sánchez, C. y López García, X. (2020). Comunicación y crisis del coronavirus en España. Primeras lecciones. El profesional de la información, 29(3), e290304. doi: 10.3145/epi.2020.may.04

Cotino Hueso, L. (2017). El reconocimiento y contenido internacional del acceso a la información pública como derecho fundamental. Teoría y Realidad Constitucional, 40, 279-316. doi: $10.5944 /$ trc.40.2017.20910

Curto Rodríguez, R. (2020). "Transparencia operativa de las comunidades autónomas españolas mediante sus portales de datos abiertos". El profesional de la información, v. 29, n. 1, e290115. https://doi.org/10.3145/epi.2020.ene.15

Díez Garrido, M. y Campos Domínguez, E. (2018). Los periodistas ante la transparencia en España. Valoración y uso de la apertura informativa. Revista Española de la Transparencia, 7, 49-69. Recuperado de https://revistatransparencia.com/ojs/index.php/ret/article/view/9

De la Sierra Morón, S. (2020). Lectura de urgencia de las reacciones frente al COVID19 desde una óptica jurídica internacional y comparada. El Cronista del Estado Social y Democrático, 86-87, 32-41. Recuperado de http://www.elcronista.es/El-Cronistan\%C3\%BAmero-86-87-Coronavirus.pdf

Espinosa Sánchez, N. (2020). Censura y manipulación informativa durante las primeras semanas de la crisis del Coronavirus en España. La Razón Histórica, 46, 39-62. Recuperado de https://www.revistalarazonhistorica.com/46-6/

Fernández Ramos, S. (2018). La transparencia pública: pasado, presente y futuro. Revista Aragonesa de Administración Pública, 51, 213-243.

García Melián, J.C. (2020). La contratación pública de emergencia. Revista de Contratación Pública Práctica, 167, 81-87.

Gamero Casado, E. (2020). Transparencia y contratación de emergencia ante I Covid19. Observatorio de Contratación Pública [entrada de fecha 16/04/2020]. http://www.obcp.es/opiniones/transparencia-y-contratacion-de-emergencia-ante-elcovid-19

Hernández Ramos, M. (2018). El derecho de acceso a la información pública en la jurisprudencia del TEDH: un derecho instrumental imprescindible para la eficacia del 
Periodismo, comunicación institucional y transparencia: aprendizajes de la crisis sanitaria del COVID-19

Convenio desde la teoría general de los derechos. Teoría y Realidad Constitucional, 42, 483-509. doi: $10.5944 /$ trc.42.2018.23641

Ibáñez García, I. (2020). "Transparencia suspendida en tiempos del coronavirus" [Entrada de blog 11/04/2020]. https://hayderecho.expansion.com/2020/04/11/transparencia-suspendida-en-tiemposdel-coronavirus/

Ibáñez Peiró, A. (2020). La actividad informativa del Gobierno español durante la emergencia sanitaria provocada por el coronavirus, COVID-19. Revista Española de Comunicación en Salud, sup. 1, S304-318. doi: 10.20318/recs.2020.5441

La Rosa Barrolleta, L. y Sandoval Martín, T. (2016). La insuficiencia de la Ley de Transparencia para el ejercicio del periodismo de datos en España. Revista Latina de Comunicación Social, 71, 1208-1229. Recuperado de http://www.revistalatinacs.org/071/paper/1142/62es.html

Manfredi Sánchez, J.L. (2014). Buenas y malas noticias sobre la ley de transparencia. Cuadernos de Periodistas, 27, 72-80. Recuperado de http://www.cuadernosdeperiodistas.com/media/2014/02/72-80-JuanManfredi.pdf

Magallón Rosa, R. (2013). ¿Por qué es importante una buena ley de transparencia para el periodismo y para los periodistas?. Instituto para la Innovación Periodística (30/09/2013). Recuperado de http://www.2ip.es/wpcontent/uploads/2013/01/Ley trasnparencia.pdf

Martínez Solana, Y. (2004). La crisis de las "vacas locas" en España: la necesidad de una información sanitaria responsable. Estudios sobre el mensaje periodístico, 10, 139-158. Recuperado https://revistas.ucm.es/index.php/ESMP/article/view/ESMP0404110139A/12581

Mir Puigpelat, O. (2017). El acceso a la información pública en la legislación española de transparencia: crónica de un cambio de paradigma. Revista Catalana de Dret Públic, 55, 48-66. doi: $\underline{10.2436 / \text { rcdp.i55.2017.3018 }}$

Moretón Toquero, A. (2014). Los límites del derecho de acceso a la información pública. Revista Jurídica de Castilla y León, 33, 1-24. Recuperado de https://www.jcyl.es/web/jcyl/AdministracionPublica/es/Plantilla100Detalle/1215245063 $\underline{566 / 1215245063566 / 1284319383546 / R e d a c c i o n}$

Piñar Mañas, J.L. (2014). Transparencia y Derecho de Acceso a la Información Pública. Algunas reflexiones en torno al Derecho de Acceso en la Ley 19/2013, de transparencia, acceso a la información y Buen Gobierno. Revista Catalana de Dret Públic, 49, 1-19. doi: $10.2436 / 20.8030 .01 .29$ 
Periodismo, comunicación institucional y transparencia: aprendizajes de la crisis sanitaria del COVID-19

Ramírez Alujas, A. (2010). Gobierno Abierto, Servicios Públicos 2.0 y Ciudadanía Digital: Notas para una nueva agenda de modernización de la gestión pública en Iberoamérica. GIGAPP Estudios/Working Papers, 2011-09. Recuperado de http://www.gigapp.org/administrator/components/com jresearch/files/publications/WP -2011-09.pdf

Rubio Jiménez, M. (2017a). ¿Cómo y cuánto usamos los periodistas españoles las herramientas dispuestas por la ley de transparencia?. Cuadernos de Periodistas, 34, 33-42. Recuperado http://www.cuadernosdeperiodistas.com/media/2017/09/Mariela Rubio.pdf

Rubio Jiménez, M. (2017b). Ley de transparencia y periodismo en España: el derecho de acceso a la información pública desde el punto de vista de los profesionales de los medios de comunicación [Tesis doctoral]. Madrid: Universidad Europea de Madrid.

Sánchez de Diego, M. (2010). De la libertad de expresión al derecho de acceso a la información pública. Comunicación y Pluralismo, 9, 231-266. Recuperado de https://summa.upsa.es/viewer.vm?id=30689

Sánchez de Diego, M. (2016). El principio de transparencia y el derecho de acceso a la información pública. En Cascajo Castro, J.L. y Martín de la Vega, A., Participación, Representación y Democracia XII Congreso de la Asociación de Constitucionalistas de España. Valencia: Tirant Lo Blanch, pp. 297-318.

Sánchez de Diego, M. y Sierra Rodríguez, J. (2020). Retos para una agenda de la transparencia. En VV.AA., Transparencia y participación para un Gobierno Abierto. Madrid: Wolters Kluwer, pp. 19-46.

Villoria Mendieta, M. (2014). Desarrollo, buen gobierno y transparencia: revisión conceptual. Revista Tiempo de Paz, 114, 4-10. Recuperado de http://revistatiempodepaz.org/revista-114/\#dflip-df 1425/5/

Westlund, O. y Ghersetti, M. (2015). Modelling news media use. Positing and applying the GC/MC model to the analysis of media use in everyday life and crisis situations. Journalism studies, 16(2), 133-151. doi: 10.1080/1461670X.2013.868139

Zafra Díaz, J. M. (2013). ¿Cómo puede cambiar el panorama mediático con una ley de transparencia?. Telos: Cuaderno de comunicación e innovación, 94, 81-83. Recuperado de https://telos.fundaciontelefonica.com/archivo/numero094/comopuede-cambiar-el-panorama-mediatico-con-una-ley-de-transparencia/ 
Periodismo, comunicación institucional y transparencia: aprendizajes de la crisis sanitaria del COVID-19

\begin{abstract}
AUTOR
Javier Sierra Rodríguez

Es Doctor en Derecho y Licenciado en Ciencias Políticas. Ha ejercido como consultor durante más de 15 años con una amplia experiencia en materia de investigación social y planificación de políticas públicas. Ha sido profesor asociado de la Facultad de Derecho de la Universidad de Murcia durante más de una década, y actualmente imparte docencia en la Universidad de Alicante y en el Centro Universitario ISEN de Cartagena adscrito a la Universidad de Murcia. Ha desarrollado una intensa actividad investigadora, participando en Congresos Internacionales, contando con más de cuarenta publicaciones relacionadas con los sistemas electorales, la transparencia y la participación, además de realizar estancias en Bruselas, Budapest y Roma. Participa anualmente en la organización del Congreso Internacional de Transparencia y es el Subdirector de la Revista Española de la Transparencia. Entre 2009 y 2015 fue el Decano del Colegio Oficial de Ciencias Políticas y Sociología de la Región de Murcia.

Orcid ID: https://orcid.org/0000-0001-9002-5153

Google Scholar: https://scholar.google.es/citations?user=dUNxPP4AAAAJ\&hl=es

ResearchID: http://www.researcherid.com/rid/R-2560-2018

Academia: https://murcia.academia.edu/JavierSierraRodriguez

Researchgate: https://www.researchgate.net/profile/Javier Sierra Rodriguez

Redalyc: https://www.redalyc.org/autor.oa?id=22687
\end{abstract}

